\title{
CKM Fits: What the Data Say (Focused on B Physics)
}

\author{
S. T'Jampens \\ Laboratoire d'Annecy-le-Vieux de Physique des Particules \\ IN2P3/CNRS et Université de Savoie \\ 9, chemin de Bellevue BP 110 \\ F-74941 Annecy-le-Vieux Cedex
}

An up-to-date profile of the Cabibbo-Kobayashi-Maskawa matrix is given with emphasis on the interpretation of recent results on $\mathrm{CP}$ violation from the $\mathrm{B}$ factories. We provide a review of the relevant experimental and theoretical inputs from the contributing domains of the electroweak interaction. We give numerical and graphical constraints on the CKM parameters and predictions of related physical observables. We discuss the impact of what the data actually say on model-independent studies of new physics.

\section{The Unitary-exact Wolfenstein Parametriza- tion and Rephasing-invariant Quantities}

The Standard Model (SM) of electroweak interactions describes $\mathrm{CP}$ violation in weak interactions as a consequence of a single non-vanishing complex phase in the three-generation Cabibbo-Kobayashi-Maskawa (CKM) quark-mixing matrix [1,2]. The CKM matrix $V$ has four quantities which are fundamental constants. These may be parametrized in a variety of ways. The standard parametrization, proposed by Chau-Keung [3] and advocated by the Particle Data Group [4], uses three mixing angles $\theta_{12}, \theta_{23}, \theta_{13}$ and one $\mathrm{CP}$-odd phase $\delta_{13}$ which generates $\mathrm{CP}$ violation. The fact that there is only one independent CP-violating parameter in the electroweak sector means that this is a very predictive and testable model. Following the observation of a hierarchy between different matrix elements, Wolfenstein [5] proposed an expansion of the CKM matrix in terms of four parameters, $A, \lambda, \rho$ and $\eta$ ( $\lambda$ being the expansion parameter). Defining [6] $\lambda \equiv \sin \theta_{12}, A \lambda^{2} \equiv$ $\sin \theta_{13}$ and $A \lambda^{3}(\rho+i \eta) \equiv \sin \theta_{13} e^{-i \delta_{13}}$ to all orders in $\lambda$ (i.e., these expressions are exact by definition; they are not corrected by terms of higher order in $\lambda$ ) ensures a unitary-exact Wolfenstein parametrization. Moreover, physically meaningful quantities must be rephasing-invariant quantities. Such invariants are the moduli, $\left|V_{i j}\right|$, and the quadri-products, $V_{i j} V_{k l} V_{i l}^{*} V_{j k}^{*}$. The Wolfenstein parameters, being physical quantities, must be phase-convention invariant: $\lambda^{2}=$
$\left|V_{u s}\right|^{2} /\left(\left|V_{u d}\right|^{2}+\left|V_{u s}\right|^{2}\right), A^{2} \lambda^{4}=\left|V_{c b}\right|^{2} /\left(\left|V_{u d}\right|^{2}+\right.$ $\left.\left|V_{u s}\right|^{2}\right)$ and $\bar{\rho}+i \bar{\eta} \equiv-\left(V_{u d} V_{u b}^{*}\right) /\left(V_{c d} V_{c b}^{*}\right) . \quad \rho$ and $\eta$ with $\rho+i \eta=V_{u b}^{*} /\left(A \lambda^{3}\right)$ are not phaseconvention invariant, in contrast to $\bar{\rho}+i \bar{\eta}[7]$. The results given in this paper use the unitary-exact Wolfenstein parametrization and rephasing-invariant quantities. $\lambda$ is determined from $\left|V_{u d}\right|$ (superallowed nuclear transitions) and $\left|V_{u s}\right|$ (semileptonic kaon decays) to a combined precision of $0.5 \%$. $A$ is determined from $\left|V_{c b}\right|$ (inclusive and exclusive semileptonic $B$ decays) to a combined precision of about $2 \%$. While $\lambda$ and $A$ are well-known, the parameters $\bar{\rho}$ and $\bar{\eta}$ are much more uncertain (about $20 \%$ for $\bar{\rho}$ and $7 \%$ for $\bar{\eta}$ ). The main goal of CP-violation experiments is to over-constrain these parameters by measuring both the three angles and the sides of the unitarity triangle (UT) and, possibly, to find inconsistencies suggesting the existence of physics beyond the SM. What is important is thus the capability of the CKM mechanism to describe flavor dynamics of many constraints from vastly different scales and not the measurement of the CKM phase's value per se.

\section{CKM Metrology: Inputs to the Global Fit}

The inputs to the global fit are observables where the theoretical uncertainties are quantitatively under control in order to test the SM: $\left|V_{u d}\right|,\left|V_{u s}\right|,\left|V_{c b}\right|$ (to fix the length scale of the UT and the constraints on $A$ and $\lambda)$, and the following quantities that are sensitive to $(\bar{\rho}, \bar{\eta})$, i.e., $\left|V_{u b}\right|, \mathcal{B}(B \rightarrow \tau \nu), \varepsilon_{K}, \Delta m_{d}$, 
$\Delta m_{d} \& \Delta m_{s}, \sin 2 \beta, \cos 2 \beta, \alpha$ and $\gamma$. In this review, a frequentist statistical framework is used. The theoretical uncertainties are modeled as allowed ranges (Rfit approach) [7] and no other a priori information is assumed where none is available. This is in contrast to another approach [8] based on the Bayesian method $[9,10]$.

2.1. $\left|V_{u b}\right|$ and $\left|V_{c b}\right|$

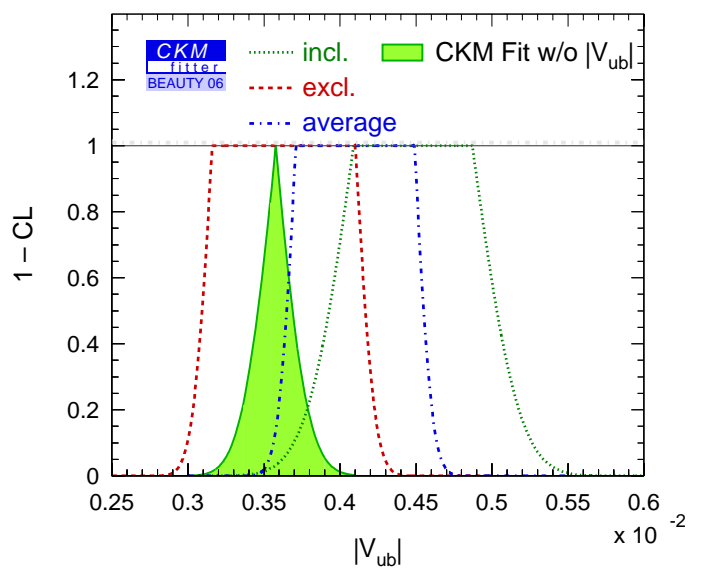

Figure 1. Confidence level as a function of $\left|V_{u b}\right|$ from the exclusive and inclusive measurements and the average. Shown in the shaded region is the prediction from the global CKM fit without including the measurements.

The magnitude of the CKM elements $V_{u b}$ and $V_{c b}$ is a critical constraint on one side of the UT. $\left|V_{u b}\right|$ is a crucial ingredient for the SM prediction of $\sin 2 \beta$. $\left|V_{c b}\right|$ is essential in the determination of the Wolfenstein parameter $A$ and plays an important role in the kaon system $\left(\varepsilon_{K}, \mathcal{B}(K \rightarrow \pi \nu \bar{\nu})\right.$, etc. $)$. Both $\left|V_{u b}\right|$ and $\left|V_{c b}\right|$ can be extracted from exclusive and inclusive semileptonic $B$ decays [11,12]. Inclusive measurements of $\left|V_{c b}\right|$ give $\left|V_{c b}\right|_{\text {inc }}=(41.7 \pm 0.7) \times 10^{-3}$ [4] with a precision of $1.7 \%$ dominated by Heavy Quark Effective (HQE) theory uncertainties, where some Operator Product Expansion (OPE) parameters are measured from the data (spectra and moments of $B \rightarrow X_{s} \gamma$ and $B \rightarrow X_{c} \ell \bar{\nu}$ distributions). The exclusive measurement $\left|V_{c b}\right|_{\text {excl }}=(39.7 \pm 2.0) \times 10^{-3}$
[13] provides an important cross check and gives a consistent result within the still large form-factor (FF) uncertainties. The extraction of $\left|V_{u b}\right|$ is more difficult because kinematic cuts are needed to suppress the large $B \rightarrow X_{c} \ell \bar{\nu}$ backgrounds. The measurement of the partial branching fractions (BR) are thus available only in a restricted phase-space region and therefore the evaluation of the theoretical uncertainties are much more difficult. Our average between the inclusive and exclusive results gives $\left|V_{u b}\right|=\left(4.10 \pm 0.09_{\exp } \pm 0.39_{\text {theo }}\right) \times 10^{-3}$. There is some tension between the CKM fit estimation of $\left|V_{u b}\right|=\left(3.59_{-0.18}^{+0.17}\right) \times 10^{-3}$ (dominated by the $\sin 2 \beta$ measurement) and the average value (pulled by the inclusive measurement) as shown in Fig. 1.

\subsection{B-meson Oscillation}

In the SM, the mass difference $\Delta m_{s}$ between the heavy and the light $B_{s}^{0}$ mass eigenstates has only a very weak dependence $\left(\mathcal{O}\left(\lambda^{2}\right)\right)$ on the Wolfenstein parameters $\bar{\rho}$ and $\bar{\eta}$. The SM prediction of $\Delta m_{q}$ $(q=d, s)$ is given by

$\Delta m_{q}=\frac{G_{F}^{2}}{6 \pi^{2}} m_{B_{q}} m_{W}^{2} \eta_{B} S_{0}\left(x_{t}\right) f_{B_{q}}^{2} B_{B_{q}}\left|V_{t q} V_{t b}^{*}\right|^{2}$,(1) where $G_{F}$ is the Fermi constant, $m_{B_{q}}$ and $m_{W}$ are the mass of the $B_{q}$ meson and $W$ boson respectively, $\eta_{B}=0.551 \pm 0.007$ is a perturbative QCD correction to the Inami-Lim function $S_{0}\left(x_{t}\right), f_{B_{q}}$ is the $B_{q^{-}}$ meson decay constant, $B_{B_{q}}$ the bag parameter and $V$ the CKM elements. A measurement of $\Delta m_{s}$ is nevertheless useful for CKM metrology within the SM, since it leads to an improvement in the constraint from the $\Delta m_{d}$ measurement on $\left|V_{t d} V_{t b}^{*}\right|^{2}$. Indeed, the ratio $\xi^{2}=\left(f_{B_{s}}^{2} B_{B_{s}}\right) /\left(f_{B_{d}}^{2} B_{B_{d}}\right)$ which quantifies $S U(3)$-breaking corrections to the hadronic matrix elements, can be calculated more accurately in lattice QCD (LQCD) than the matrix elements themselves. Hence, a measurement of $\Delta m_{s}$ reduces the uncertainty on $f_{B_{d}} \sqrt{B_{B_{d}}}$. Evidences on $\Delta m_{s}$ were reported this winter by both the D0 [15] and CDF collaborations. The latter recently reported the first observation with a significance $>5 \sigma$ [16]: $\Delta m_{s}=$ $17.77 \pm 0.10$ (stat) \pm 0.07 (syst) $\mathrm{ps}^{-1}$ with a relative uncertainty of $0.7 \%$, already smaller than for $\Delta m_{d}$ $(0.8 \%)$. The value of $\Delta m_{s}$ is in agreement with the prediction from the global CKM fit, $\Delta m_{s}=$ $18.9_{-2.8}^{+5.7} \mathrm{ps}^{-1}$ obtained without using the measurement in the fit. 
2.3. $B \rightarrow \tau \nu$

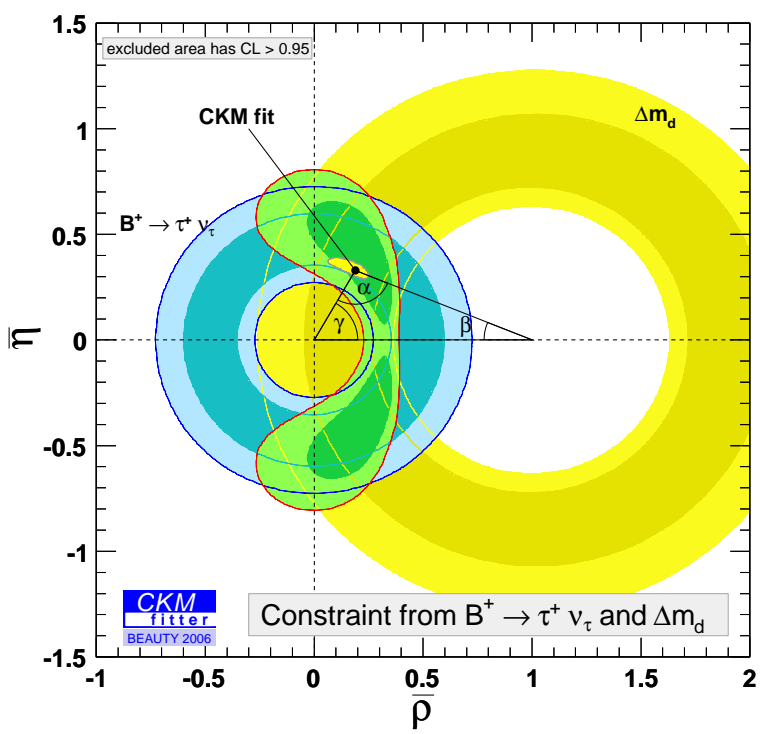

Figure 2. Constraints of $95 \% \mathrm{CL}$ in the $(\bar{\rho}, \bar{\eta})$ plane from the $B^{+} \rightarrow \tau^{+} \nu$ branching fraction, $\Delta m_{d}$, and the combined use of these (darker shades indicate $68 \%$ C.L. regions). The results are compared with the global CKM fit.

The $B^{+} \rightarrow \tau^{+} \nu$ helicity-suppressed annihilation decay is sensitive to $f_{B_{d}}\left|V_{u b}\right|$, where $\left|V_{u b}\right|$ the magnitude of the quark-mixing element. Although this decay comes from a tree diagram, it is sensitive to physics beyond the SM such as supersymmetry or two-Higgs doublet models through the introduction of a charged Higgs replacing the $W$ propagator. Both $B A B A R$ and Belle have updated their measurements [14]: $\mathcal{B}=\left(0.88_{-0.67}^{+0.68}\right.$ (stat) \pm 0.11 (syst) $) \times 10^{-4}$ for the BABAR analysis using $288 \mathrm{fb}^{-1}$ of data and $\mathcal{B}=\left(1.79_{-0.49}^{+0.56}(\text { stat })_{-0.51}^{+0.46}\right.$ (syst) $) \times 10^{-4}$ for the Belle analysis using $414 \mathrm{fb}^{-1}$ of data. The CKM fit prediction (not including the direct measurements) is $\left(0.87_{-0.20}^{+0.13}\right) \times 10^{-4}$ in good agreement with the direct measurements. $\mathcal{B}\left(B^{+} \rightarrow \tau^{+} \nu\right)$ will become with more statistics a powerful input together with $\Delta m_{d}$ : the ratio determines $\left|V_{u b}\right| /\left|V_{t d}\right|$ independent of $f_{B_{d}}$ (but dependent on the bag factor $B_{B_{d}}$ ). The allowed regions in the $(\bar{\rho}, \bar{\eta})$ plane are shown in Fig. 2. The remaining theoretical uncertainty stems from $B_{B_{d}}$ that enters the SM prediction of $\Delta m_{d}$.

\subsection{UT Angle $\beta$}

Measurements of $C P$ asymmetries in the propertime distribution of neutral $B$ decays to a common final state, $f$, provide direct information on the angles of the UT. The asymmetry reads:

$$
\begin{aligned}
a_{f} & =\frac{\Gamma\left(\bar{B}^{0}(t) \rightarrow f\right)-\Gamma\left(B^{0}(t) \rightarrow f\right)}{\Gamma\left(\bar{B}^{0}(t) \rightarrow f\right)+\Gamma\left(B^{0}(t) \rightarrow f\right)} \\
& =S_{f} \sin (\Delta m t)-C_{f} \cos (\Delta m t),
\end{aligned}
$$

with $S=2 \operatorname{Im} \lambda_{f} /\left(1+\left|\lambda_{f}\right|^{2}\right), C=\left(1-\left|\lambda_{f}\right|^{2}\right) /(1+$ $\left.\left|\lambda_{f}\right|^{2}\right)$ and $\lambda_{f}=(q / p)\left(\bar{A}_{f} / A_{f}\right) \cdot q / p$ are the $B^{0}-\bar{B}^{0}$ mixing parameters and $A_{f}\left(\bar{A}_{f}\right)$ is the amplitude of $B^{0}\left(\bar{B}^{0}\right) \rightarrow f$. The color-suppressed $\bar{b} \rightarrow \bar{c} c \bar{s}$ decays to $\mathrm{CP}$ eigenstates, like $B^{0} \rightarrow$ charmonium + $K^{0}$ modes, are the theoretically cleanest modes because they are dominated by a single weak phase. The only term with a different weak phase is a penguin contribution that is doubly Cabibbo suppressed. The asymmetry is thus, to a very good accuracy, related to $\sin 2 \beta: a_{f}=-\eta_{f} \sin 2 \beta \sin (\Delta m t)$, where $\eta_{f}$ is the CP eigenvalue of $f$. The world average is $\sin 2 \beta=0.676 \pm 0.026 \quad$ [13] including the updated measurements done by the BABAR and Belle collaborations. One angle $\beta$ among the four-fold ambiguity, $(21.2 \pm 1.0)^{\circ}$, is compatible with the result from the global CKM fit without the measurement of $\beta$, $\left(27.7_{-3.9}^{+0.8}\right)^{\circ}$. The slight shift between the measurement and the prediction from the global fit is a manifestation of the tension in $\left|V_{u b}\right|$. The measurement of the sign of $\cos 2 \beta$ helps removing two of the solutions [18]. The penguin "pollution" has been estimated to be of the order of $10^{-3}$ [19].

The $\bar{b} \rightarrow \bar{s} q \bar{q}$ penguin-dominated decays have the same weak phase as the $\bar{b} \rightarrow \bar{c} c \bar{s}$ amplitude up to small corrections (at most $\mathcal{O}(0.1)$ ) from subleading u-quark penguin diagrams leading to an effective angle $\beta_{\text {eff }}$. In $\bar{b} \rightarrow \bar{s} u \bar{u}$ decays there are also colorsuppressed tree amplitudes. Since penguin loop contributions are sensitive to physics beyond the SM, it is important to have an unambiguous estimate of the deviation $\Delta S \equiv S_{b \rightarrow s q \bar{q}}-S_{b \rightarrow c \bar{c} s}$ in the SM [20] to 
claim that any deviation from the SM is due to New Physics (NP). Various estimates, using different theoretical approaches such as QCDF, pQCD and SCET, find a small value and a positive sign for $\Delta S$ whereas the various modes measured by $B A B A R$ and Belle are all below but consistent with $S_{b \rightarrow c \bar{c} s}$ [21]. NP can affect differently the various $s$-penguin modes. A disagreement would falsify the SM. However, the interference between the SM and NP amplitudes would introduce hadronic uncertainties. As a result, the determination of the NP parameters could not be estimated in a clean way. More statistics will be needed for a mode-by-mode study.

\subsection{UT Angle $\alpha$}

The measurement of the angle $\alpha$ is an independent test of $\pi-(\beta+\gamma)$. The angle $\alpha$ is measured through the interference of $b \rightarrow u$ (tree) quark-level decays with and without mixing. However, the $b \rightarrow d$ (penguin) amplitude could be sizeable (its magnitude is of the same order in $\lambda$ as the tree amplitude) and thus leads to an effective angle $\alpha_{\text {eff }}$ and direct $\mathrm{CP}$ violation in the time-dependent $\mathrm{CP}$ asymmetry (see Eq. 2). The magnitude and relative phase of the penguin contribution to the asymmetry may be unraveled with an isospin analysis [22] among the amplitudes of $B^{0} \rightarrow h^{+} h^{-}, B^{+} \rightarrow h^{+} h^{0}$ and $B^{0} \rightarrow h^{0} h^{0}$ decays ( $h=\pi, \rho$ ) or a Dalitz-plot analysis [23] for the $\rho \pi$ decays. The isospin analysis allows to resolve $\alpha$ up to an eight-fold ambiguity within $[0, \pi]$. The isospinbreaking effects have been estimated in [24,25] and are smaller than the current precision on $\alpha$. Neglecting the electroweak penguin in $B \rightarrow \pi \pi$ amounts to a shift of $\alpha$ by $-2.1^{\circ}$ [7].

\subsection{1. $B \rightarrow \pi \pi$}

The isospin analysis uses the world average [13] $S_{\pi^{+} \pi^{-}}=-0.59 \pm 0.09, C_{\pi^{+} \pi^{-}}=-0.39 \pm 0.07$, the BRs of all three modes, and the direct $\mathrm{CP}$ asymmetry $C_{\pi^{0} \pi^{0}}=0.36_{-0.31}^{+0.33}$. It leads to two relations (by neglecting the electroweak penguin in $A^{+0}$ ): $A^{+0}=$ $A^{+-} / \sqrt{2}+A^{00}$, and a similar expression for the $\bar{A}$ 's (CP-conjugate amplitudes). These relations can be represented as triangles in the complex plane. There is a disagreement between the BABAR and Belle results on $C_{\pi^{+} \pi^{-}}$of $2.3 \sigma$. Taking properly into account final-state radiative corrections in order to determine the non-radiative BRs [26] is also important: recently, the BABAR collaboration [27] showed a 3 to $6 \%$ shift effect in the BR values. The most difficult measurement comes from the $\pi^{0} \pi^{0}$ modes, in particular in the need to determine CP-tagged rates to measure $C_{\pi^{0} \pi^{0}}$. These difficulties lead to the development of bounds on $\Delta \alpha \equiv \alpha-\alpha_{\text {eff }}$ [28]. The isospin analysis done without $C_{\pi^{0} \pi^{0}}$ leads to the same results on $\Delta \alpha$ as done by applying the optimal bounds. $S_{\pi^{0} \pi^{0}}$, which requires a time-dependent analysis, will not be available in the near future (it would help resolving ambiguities). Because the BR for $B^{0} \rightarrow \pi^{0} \pi^{0}$ is not much smaller than the $B^{+} \rightarrow \pi^{+} \pi^{0}$ and $B^{0} \rightarrow \pi^{+} \pi^{-}$ channels, the limit on $\left|\Delta \alpha_{\pi \pi}\right|$ is weak: $\left|\Delta \alpha_{\pi \pi}\right|<$ $32.1^{\circ}$ at $95 \%$ confidence level (CL). Future improvement will almost entirely come from $C_{\pi^{0} \pi^{0}}$.

2.5.2. $B \rightarrow \rho \rho$

The $\rho \rho$ channels are more complicated than $\pi \pi$ because of their vector-vector nature which implies a mixture of CP-even and CP-odd components. The isospin analysis can be applied to each polarization state but the fact that the measured longitudinal polarization is close to unity greatly simplifies the analysis since the CP-even fraction dominates. The BABAR collaboration found $3 \sigma$ evidence [29] with a sample of 348 millions $B \bar{B}$ decays for $B^{0} \rightarrow \rho^{0} \rho^{0}$ (no evidence yet from the Belle collaboration) allowing for the first time a full isospin analysis. They measure $\mathcal{B}=$ $\left(1.16_{-0.36}^{+0.37}\right.$ (stat) \pm 0.27 (syst) $) \times 10^{-6}$ and a longitudinal fraction $f_{L}=0.86_{-0.13}^{+0.11}$ (stat) \pm 0.05 (syst). In contrast to the $\pi \pi$ channels, the $B^{0} \rightarrow \rho^{0} \rho^{0}$ decay has a much smaller BR than the $B^{0} \rightarrow \rho^{+} \rho^{-}$and $B^{+} \rightarrow \rho^{+} \rho^{0}$ channels. As a consequence, it is possible to set a tighter limit on $\Delta \alpha_{\rho \rho}:\left|\Delta \alpha_{\rho \rho}\right|<22.4^{\circ}$ at $95 \% \mathrm{CL}$. The isospin analysis using the world average $S_{\rho \rho}=-0.13 \pm 0.19, C_{\rho \rho}=-0.06 \pm 0.14$ and the BRs of all three modes (using the world average for $\rho^{+} \rho^{-}$and $\rho^{+} \rho^{0}$ and the $B A B A R$ result for $\left.\rho^{0} \rho^{0}\right)$ gives $\alpha=(94 \pm 21)^{\circ}$ with a mirror solution at $3 \pi / 2-\alpha$. At present, the complication such as the $\rho-\omega$ mixing or the finite $\rho$ width allowing a $I=1$ isospin state [30] can be neglected.

\subsection{3. $B \rightarrow \rho \pi$}

Unlike $\pi^{+} \pi^{-}$and $\rho^{+} \rho^{-}, \rho^{ \pm} \pi^{\mp}$ is not a CP eigenstate, leading to the need to consider four flavorcharge configurations. The corresponding isospin analysis in the quasi-two-body approximation (Q2B) 
is more complicated since two pentagonal amplitude relations [31] with 12 unknowns must be solved (to be compared with the triangular amplitude relations with 6 unknowns for the $\pi \pi$ and $\rho \rho$ channels). The timedependent Dalitz-plot analysis of $B^{0} \rightarrow \pi^{+} \pi^{-} \pi^{0}$ in the Q2B approximation, dominated by the $\rho(770)$ channels and their interferences, allows a simultaneous extraction of $\alpha$ and the strong phases as pointed out by Snyder and Quinn [23]. The interference regions of the $\rho^{+} \pi^{-}, \rho^{-} \pi^{+}$and $\rho^{0} \pi^{0}$ amplitudes in the Dalitz plot introduce a dependence on $\cos 2 \alpha$ as well as $\sin 2 \alpha$. Therefore, a single discrete ambiguity remains $(\alpha \rightarrow \alpha+\pi)$. There are a number of theoretical and experimental complications in this analysis like higher resonances, the $\rho$ shape uncertainties, non-resonant $3 \pi$ backgrounds [32], electroweak penguins [33], etc. The analysis is performed by measuring 26 bilinear FF coefficients [34]. The physical quantities are extracted by a correlated $\chi^{2}$ fit. Both $B A B A R$ and Belle have updated their measurements [35]. Belle also performed a combined Dalitz and pentagonal isospin analysis by using the world average BRs and asymmetries [13]: $\mathcal{B}\left(B^{0} \rightarrow \rho^{ \pm} \pi^{\mp}\right)$, $\mathcal{B}\left(B^{+} \rightarrow \rho^{+} \pi^{0}\right), \mathcal{B}\left(B^{+} \rightarrow \rho^{0} \pi^{+}\right), \mathcal{A}\left(B^{+} \rightarrow \rho^{+} \pi^{0}\right)$ and $\mathcal{A}\left(B^{+} \rightarrow \rho^{0} \pi^{+}\right)$. The isospin analysis alone can determine $\alpha$ up to multiple discrete ambiguities [36]. Although the constraints are moderate so far (no constraint at a $95 \% \mathrm{CL}$ on $\alpha$ is obtained), it helps singling out with $B \rightarrow \pi \pi, \rho \rho$ the value of $\alpha$ compatible with the CKM fit (see Fig. 3).

\subsection{4. combined analysis}

A combined analysis of the $\pi \pi, \rho \rho$ and $\rho \pi$ systems gives $\alpha=\left(93_{-9}^{+11}\right)^{\circ}$ (see Fig. 3). This measurement is in agreement with the CKM fit expectation $\alpha=$ $\left(100_{-7}^{+5}\right)^{\circ}$. With more statistics, many assumptions (Q2B approximation, Breit-wigner shape, large-finite $\rho$ width, non-resonant background, isospin-breaking effects, etc.) will need a closer look [37] in the extraction of $B \rightarrow \rho \pi(B \rightarrow \rho \rho)$ from the dynamics of $B \rightarrow 3 \pi(B \rightarrow 4 \pi)$ final states.

\subsection{UT Angle $\gamma$}

The angle $\gamma$ is measured through the interference of tree-level $\bar{b} \rightarrow \bar{c} u \bar{s}\left(B^{+} \rightarrow D^{(*) 0} K^{+}\right)$and $\bar{b} \rightarrow \bar{u} c \bar{s}$ $\left(B^{+} \rightarrow \bar{D}^{(*) 0} K^{+}\right)$amplitudes, where the $D$ and $\bar{D}$ decay into the same final state. It is thus not theory limited (even if $D^{0}-\bar{D}^{0}$ mixing has not yet been seen,

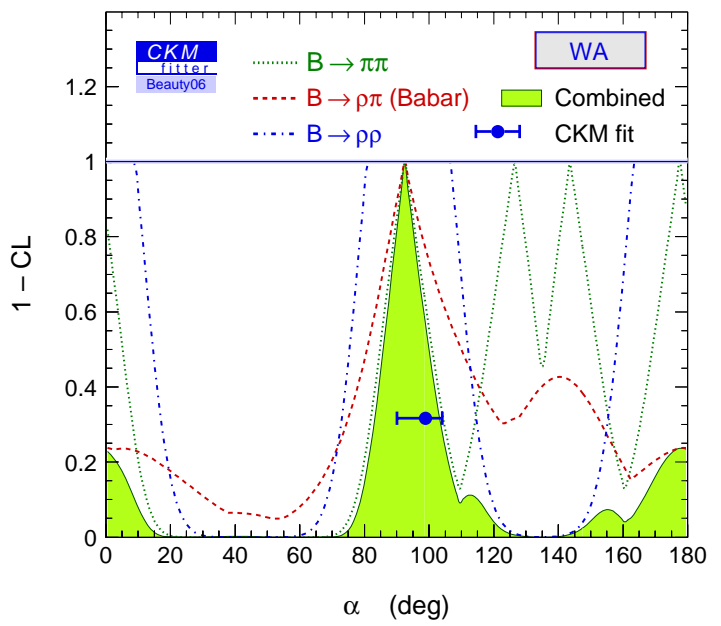

Figure 3. Confidence level as a function of the angle $\alpha$ extracted from an isospin analysis of the world averages for $B \rightarrow \pi \pi$ and $B \rightarrow \rho \rho$ and the BABAR measurement for $B \rightarrow \rho \pi$ (Snyder-Quinn Dalitz method). The shaded region is the combination. Also shown is the prediction of the CKM fit (not including the direct measurements).

the theory error on $\gamma$ is conservatively lower than $10^{-5}$ [25]) and an important ingredient in the reference UT, the unitarity triangle obtained from tree-only decays (no penguin "pollution") and thus unlikely to be affected by NP. Several variants were proposed to measure $\gamma$ which can be grouped by the choice of the $D$ decays: CP eigenstate, e.g., $K_{S}^{0} \pi^{0}$ (GLW) [38], flavor-specific state, e.g., $K^{-} \pi^{+}$(ADS) [39] and the many-body final state (interference in the Dalitz plot), e.g., $K_{S}^{0} \pi^{+} \pi^{-}$(GGSZ) [40]. All methods fit simultaneously $\gamma$, the strong phases $\delta$ and the ratio of the suppressed-to-dominated amplitudes $r_{B}$ ( $\gamma$ is common to all final states whereas $\delta$ and $r_{B}$ depend on the final states). A naïve average of $\gamma$ 's leads to an underestimated uncertainty on $\gamma$ because of the nuisance parameters [41] $\delta$ and $r_{B}$. One needs to perform a full global fit with all the inputs from the various decays to extract correctly $\gamma$. The crucial parameter is the size of the $r_{B}$ parameter because the uncertainty on $\gamma$ scales roughly like $1 / r_{B}$. ( $\gamma$ is the phase of a complex number whose modulus is $r_{B}$ thus the smaller 


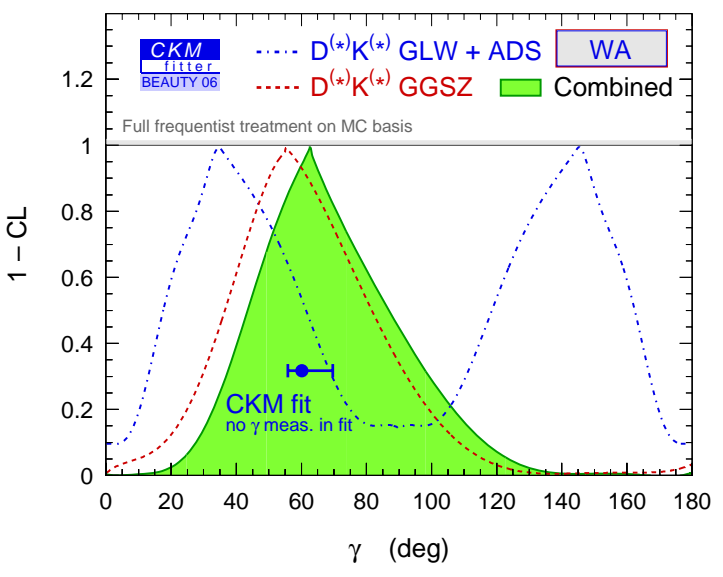

Figure 4. Confidence level as a function of the angle $\gamma$ from the GLW, ADS and GGSZ methods. Also shown is the prediction of the CKM fit.

the $r_{B}$, the larger the error on $\gamma$ ). Combining the GLW, ADS and GGSZ analyses using a frequentist statistical framework results in $\gamma=\left(60_{-24}^{+38}\right)^{\circ}$ (see Fig. 4) and in the average $r_{B}$ values of all modes: $r_{B}(D K)=0.10_{-0.04}^{+0.03}, r_{B}\left(D^{*} K\right)=0.10_{-0.06}^{+0.04}$ and $r_{B}\left(D K^{*}\right)=0.11_{-0.11}^{+0.09}$. The prediction of the SM CKM fit gives $\gamma=\left(59_{-4}^{+9}\right)^{\circ}$ in agreement with the overall $\gamma$ average. It will take more data to pin down $r_{B}$ and determine $\gamma$ more precisely.

\section{Global Fit}

The unitarity triangle checks for the consistency of the information obtained from mixing with the information obtained from decay. This is only one of many tests of the CKM matrix. The strategy of placing all CKM constraints on the $(\bar{\rho}, \bar{\eta})$ plane is a convenient way to compare the overconstraining measurements and a way to search for NP by looking for inconsistencies. The $95 \% \mathrm{CL}$ of the individual constraints and the result of the global fit are displayed in Fig. 5. For the detailed inputs, see [7]. The fit output is given in Table 1. The $\gamma$ and $\alpha$ measurements together with $\left|V_{u b} / V_{c b}\right|$ determine $\bar{\rho}$ and $\bar{\eta}$ from (effectively) tree-level processes, independent of mixing, and agree with the other loop-induced constraints. Present CKM fits provide a consistency check of the

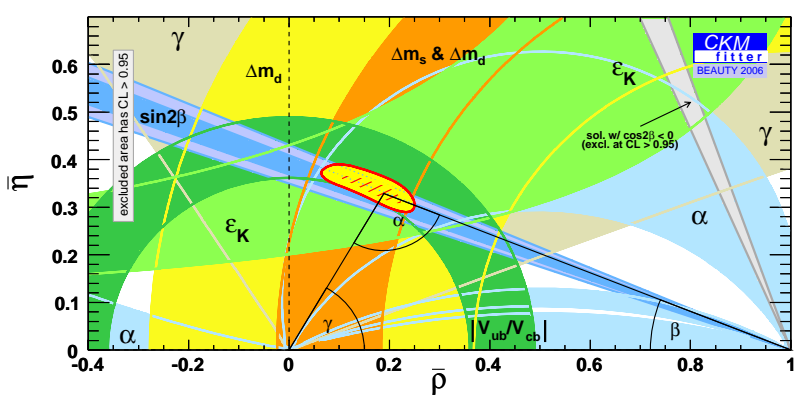

Figure 5. Individual constraints and the global CKM fit on the $(\bar{\rho}, \bar{\eta})$ plane. The shaded areas have $95 \%$ CL.

SM hypothesis but do not provide a bound on NP parameter space. The rather large allowed regions provided by each constraint individually (but the UT angles), are mainly due to theoretical uncertainties obtained with LQCD.

\section{Model-independent Constraints on New Physics in $|\Delta B|=2$ Transitions}

In this section, we allow for new phases to be present in the $B_{q}^{0}-\bar{B}_{q}^{0}(q=d, s)$ mixing but we assume that the tree-level decay amplitudes are given by the SM diagrams and that the $3 \times 3 \mathrm{CKM}$ matrix is unitary. This framework includes a large class of NP models. NP can be parametrized as $M_{12}^{q}=$ $\left(M_{12}^{q}\right)^{S M}\left(1+h_{q} e^{i 2 \sigma_{q}}\right)=\left(M_{12}^{q}\right)^{S M} r_{q}^{2} e^{i 2 \theta_{q}}$ where $M_{12}^{q}$ is the dispersive part of the $B_{q}^{0}-\bar{B}_{q}^{0}$ mixing amplitude (the superscript SM stands for the mixing amplitude in the SM) and $h_{q}$ and $\sigma_{q}$ are the magnitude and phase, respectively, introduced by the NP amplitude. $B_{q}^{0}-\bar{B}_{q}^{0}$-mixing-dependent observables sensitive to NP are $\Delta m_{q}, S_{f}, A_{S L}^{q}, \Delta \Gamma_{q}$. These observables are modified compared to the SM ones as follows:

$$
\begin{aligned}
\Delta m_{q} & =\Delta m_{q}^{S M} r_{q}^{2}, \\
S_{\psi K} & =\sin \left(2 \beta+2 \theta_{d}\right), \\
S_{\psi \phi} & =\sin \left(2\left|\beta_{s}\right|-2 \theta_{s}\right), \\
A_{S L}^{q} & =\operatorname{Im}\left\{\Gamma_{12}^{q} /\left(\left(M_{12}^{q}\right)^{S M} r_{q}^{2} e^{i 2 \theta_{q}}\right\},\right. \\
\Delta \Gamma_{s}^{C P} & =\left(\Delta \Gamma_{s}^{C P}\right)^{S M} \cos ^{2}\left(2\left|\beta_{s}\right|-2 \theta_{s}\right),
\end{aligned}
$$


Table 1

Global CKM fit results.

\begin{tabular}{lcc}
\hline Observable & central $\pm \mathrm{CL} \equiv 1 \sigma$ & $\pm \mathrm{CL} \equiv 2 \sigma$ \\
\hline$\lambda$ & $0.22717_{-0.00101}^{+0.00100}$ & ${ }_{-0.00202}^{+0.00200}$ \\
$A$ & $0.806_{-0.014}^{+0.014}$ & ${ }_{-0.028}^{+0.029}$ \\
$\bar{\rho}$ & $0.195_{-0.055}^{+0.022}$ & ${ }_{-0.110}^{+0.044}$ \\
$\bar{\eta}$ & $0.326_{-0.015}^{+0.027}$ & ${ }_{-0.030}^{+0.053}$ \\
\hline$J\left[10^{-5}\right]$ & $2.91_{-0.14}^{+0.25}$ & ${ }_{-0.28}^{+0.51}$ \\
\hline$R_{u}$ & $0.3798_{-0.0090}^{+0.0107}$ & ${ }_{-0.0182}^{+0.0256}$ \\
$R_{t}$ & $0.868_{-0.025}^{+0.060}$ & ${ }_{-0.049}^{+0.118}$ \\
\hline$\alpha(\mathrm{deg})$ & $99.0_{-9.4}^{+4.0}$ & ${ }_{-17.9}^{+8.0}$ \\
$\beta(\mathrm{deg})$ & $22.03_{-0.62}^{+0.72}$ & ${ }_{-1.27}^{+1.69}$ \\
$\gamma(\mathrm{deg})$ & $59.0_{-3.7}^{+9.2}$ & ${ }_{-7.3}^{+18.0}$ \\
\hline$\left|V_{u d}\right|$ & $0.97385_{-0.00023}^{+0.00024}$ & ${ }_{-0.00047}^{+0.00047}$ \\
$\left|V_{u s}\right|$ & $0.22715_{-0.00100}^{+0.00101}$ & ${ }_{-0.00201}^{+0.00201}$ \\
$\left|V_{u b}\right|\left[10^{-3}\right]$ & $3.683_{-0.079}^{+0.106}$ & ${ }_{-0.158}^{+0.263}$ \\
$\left|V_{c d}\right|$ & $0.22703_{-0.00100}^{+0.00102}$ & ${ }_{-0.00201}^{+0.00202}$ \\
$\left|V_{c s}\right|$ & $0.97299_{-0.00023}^{+0.00024}$ & ${ }_{-0.00047}^{+0.00047}$ \\
$\left|V_{c b}\right|\left[10^{-3}\right]$ & $41.61_{-0.63}^{+0.62}$ & ${ }_{-1.25}^{+1.24}$ \\
$\left|V_{t d}\right|\left[10^{-3}\right]$ & $8.20_{-0.27}^{+0.59}$ & ${ }_{-0.53}^{+1.18}$ \\
$\left|V_{t s}\right|\left[10^{-3}\right]$ & $40.96_{-0.62}^{+0.60}$ & ${ }_{-1.23}^{+1.21}$ \\
$\left|V_{t b}\right|$ & $0.999127_{-0.000026}^{+0.000026}$ & ${ }_{-0.000052}^{+0.000052}$ \\
\hline & &
\end{tabular}

where $S_{f}$ are the time-dependent $\mathrm{CP}$ asymmetries, $\beta_{s}=\arg \left[-\left(V_{c s} V_{c b}^{*}\right) /\left(V_{t s} V_{t b}^{*}\right)\right] \approx-1^{\circ}, A_{S L}^{q}$ the $\mathrm{CP}$ asymmetries in the semileptonic decays (although unimportant in the global fit, play a role in constraining such extensions of the SM [42]) and $\Delta \Gamma_{s}^{C P}$ the lifetime difference between the CP-even and CP-odd $B_{s}$ state. NP contributions reduce $\Delta \Gamma$ and enhance the mixing frequency $\Delta m_{q}$ with respect to the SM ones.

The UT of the $B_{d}$ system being non-squashed implies that the determination of the NP parameters $\left(h_{d}, \sigma_{d}\right)$ are strongly correlated to $(\bar{\rho}, \bar{\eta})$. On the contrary, the UT of the $B_{s}$ system is highly squashed: the determination of NP parameters $\left(h_{s}, \sigma_{s}\right)$ are almost independent of $(\bar{\rho}, \bar{\eta})$. Not all CP-violating measurements can be interpreted as constraints on the $(\bar{\rho}, \bar{\eta})$ plane. For example, the asymmetry in $B_{s}^{0} \rightarrow J / \psi \phi$ which measures $\sin \left(2\left|\beta_{s}\right|-2 \theta_{s}\right)$ is a perfect channel to look for NP in $B_{s}^{0}-\bar{B}_{s}^{0}$ mixing since the SM expectation of this asymmetry is small and well-predicted, $\sin 2 \beta_{s}=-0.035_{-0.002}^{+0.003}$. Other rare $\Delta B=1$ FlavorChanging Neutral-Current (FCNC) $B$ decays (e.g., $\left.B \rightarrow X_{s} \gamma, B \rightarrow X_{s} \ell^{+} \ell^{-}, B_{d, s} \rightarrow \ell^{+} \ell^{-}\right)$are also important. These theoretically-clean measurements will be an essential ingredient in understanding the NP flavor structure.

While the constraints on the $B_{d}$ NP parameters are significant, NP with a generic phase may still contribute to $M_{12}^{d}$ at the order of $30 \% . r_{d}^{2}=1.01_{-0.41}^{+0.52}$ and $2 \theta_{d}=-0.036_{-0.145}^{+0.035}$ where the slight shift compared to the SM values $\left(r_{d}^{2}=1,2 \theta=0\right)$ is due to the tension between $\left|V_{u b}\right|$ and the $\sin \left(2 \beta+2 \theta_{d}\right)$ measurement.

Compared to the $B_{d}$ sector, the $B_{s}$ is less well known. Although the $\Delta m_{s}$ measurement indicates a SM-like $B_{s}^{0}-\bar{B}_{s}^{0}$ mixing, no significant information is available on the $B_{s}^{0}-\bar{B}_{s}^{0}$ mixing phase, $\beta_{s}$, and the width difference $\Delta \Gamma_{s}$ has large uncertainties. $\Delta m_{s}$, $\Delta \Gamma_{s}$ and $A_{S L}^{s}$ allow first constraints in the $B_{s}$ sector. The bounds on the $B_{s}$ NP parameters are weaker than on the $B_{d}$ ones since the only available measurement, $\Delta m_{s}$, suffers from larger theoretical uncertainties. Only progress in LQCD will help to improve this constraint (the situation is the same in the $B_{d}$ sector but significant constraints come from (effectively) tree-level measurements). $\Delta \Gamma_{s}$ [13] gives significant constraints, despite its poor knowledge because its central value disfavors any deviation from the SM. Once more precisely known from LHC data, the theoretical uncertainties will dominate.

\section{Conclusion}

The CKM mechanism has been very successful in describing flavor dynamics of many observables sensitive to vastly different scales. The improvement in LQCD is crucial to further constrain the $(\bar{\rho}, \bar{\eta})$ plane to seek for inconsistencies. After the many great measurements done at the $B$ factories and the Tevatron experiments, the near future will bring many new re- 
sults, either with better accuracy or measurements yet to be done to seek deviations from the SM. It is important to make sure that everything is well-controlled (assumptions, theoretical uncertainties, etc.) before claiming a NP discovery.

\section{REFERENCES}

1. N. Cabibbo, Phys. Rev. Lett. 51, (1963) 531.

2. M. Kobayashi and T. Maskawa, Prog. Theor. Phys. 49, (1973) 652.

3. L.L. Chau and W.Y. Keung, Phys. Rev. Lett. 53. (1984) 1802.

4. Particle Data Group, W.-M. Yao et al., Jour. Phys. G 33, (2006) 1 .

5. L. Wolfenstein, Phys. Rev. Lett. 51, (1983) 1945.

6. A.J. Buras et al., Phys. Rev. D 50, (1994) 3433.

7. CKMfitter group (J. Charles et al.), Eur. Phys. Jour. C 41, (2005) 1., updated in http://ckmfitter.in2p3.fr

8. UTfit collaboration (M. Bona et al.), Jour. High En. Phys. 0507, (2005) 28, updated in http://www.utfit.org

9. D.R. Cox, Principle of Statistical Inference, Cambridge University Press (2006). F. James, Statistical Methods in Experimental Physics, World Scientific Press (2006).

10. J. Charles et al., hep-ph/0607246

11. L. Gibbons, these proceedings

12. G. Paz, these proceedings

13. Heavy Flavor Averaging Group, http://www.slac.stanford.edu/xorg/hfag

14. BABAR collaboration (B. Aubert et al.), hepex/0608019; Belle collaboration (K. Ikado et al.), hep-ex/0604018

15. D0 collaboration (V. Abazov et al.), hepex/0603029; CDF collaboration (A. Abulencia $e t$ $a l$. ), hep-ex/0606027

16. CDF collaboration (A. Abulencia et al.), hepex/0609040

17. M. Okamoto, Proc. Sci. LAT2005 (2005) [heplat/0510113]

18. H. Lacker, these proceedings

19. H. Boos et al., Phys. Rev. D 70 (2004) 036006; M. Ciuchini et al., Phys. Rev. Lett. 95, (2005) 21804; H.-n Li and S. Mishima, hep-ph/0610120

20. C.-k Chua, hep-ph/0605301 and references therein
21. Y. Ushiruda, these proceedings

22. M. Gronau and D. London, Phys. Rev. Lett. 65, (1990) 3381.

23. H.R. Quinn and A.E. Snyder, Phys. Rev. D 48, 2139 (1993) 2139.

24. M. Gronau and J. Zupan, Phys. Rev. D 71, (2005) 074017; S. Gardner, Phys. Rev. D 72, (2005) 034015 .

25. J. Zupan, these proceedings

26. E. Baracchini and G. Isidori, Phys. Lett. B 633, (2006) 309.

27. BABAR collaboration (B. Aubert et al.), hepex/0608003

28. J. Charles, Phys. Rev. D 59, (1999) 054007; M. Gronau et al., Phys. Lett. B 514, (2001) 315.

29. BABAR collaboration (B. Aubert et al.), hepex/0607097

30. A. Falk et al., Phys. Rev. D 69, (2004) 011502.

31. H.J. Lipkin et al., Phys. Rev. D 44, (1991) 1454.

32. J. Tandeau and S. Gardner, hep-ph0204147

33. J. Charles, PhD thesis, Université Paris Sud (1999)

34. H.R. Quinn and J.P. Silva, Phys. Rev. D 62, (2000) 054002.

35. BABAR collaboration (B. Aubert et al.), hepex/0608002; Belle collaboration (K. Abe et al.), hep-ex/0609003

36. M. Gronau, Phys. Lett. B 265, (1991) 389.

37. M. Gonau and J. Zupan, Phys. Rev. D 71, (2005) 074017.

38. D. Atwood et al., Phys. Rev. Lett. 78, (1997) 3257; Phys. Rev. D 63, (2001) 036005.

39. M. Gronau and D. London, Phys. Lett. B 253, (1991) 483; M. Gronau and D. Wyler, Phys. Lett. B 265, (1991) 172.

40. A. Giri et al., Phys. Rev. D 68, (2003) 054018.

41. R.D. Cousins, Proc. PHYSTAT05 (2006).

42. S. Laplace et al., Phys. Rev. D 65, (2002) 094004. 